\title{
Thalamocortical Asynchrony in Conditions of Spinal Cord Injury Pain in Rats
}

\author{
David A. Seminowicz, ${ }^{1,2}$ Li Jiang, ${ }^{2,3}$ Yadong Ji, ${ }^{3}$ Su Xu, ${ }^{5,6}$ Rao P. Gullapalli, ${ }^{5,6}$ and Radi Masri ${ }^{2,3,4}$ \\ ${ }^{1}$ Department of Neural and Pain Sciences, ${ }^{2}$ Program in Neuroscience, and ${ }^{3}$ Department of Endodontics, Prosthodontics and Operative Dentistry, University \\ of Maryland School of Dentistry, and ${ }^{4}$ Department of Anatomy and Neurobiology, ${ }^{5}$ Department of Sciences of Diagnostic Radiology and Nuclear Medicine, \\ and ${ }^{6}$ Core for Translational Research in Imaging @ Maryland, University of Maryland School of Medicine, Baltimore, Maryland 21201
}

\begin{abstract}
Spinal cord injury (SCI) pain is a debilitating chronic condition that is severe and unrelenting. Despite decades of extensive research, the neuropathological mechanisms responsible for the development of this devastating condition remain largely unknown, hindering our ability to develop effective treatments. Because several lines of evidence implicate abnormalities of the thalamus and cortex in the etiology of SCI pain, we hypothesized that SCI pain results from abnormal functional connectivity of brain areas heavily implicated in pain processing. We performed a longitudinal study in a rat model of SCI (SCI group, $n=8$; sham-operated group, $n=6$ ) and acquired resting-state functional magnetic resonance imaging scans before spinal surgery and 3, 7, 14, and 21 (SCI only) days after surgery in the same animals. Functional connectivity was decreased between the ventroposterior lateral thalamus (VPL) and primary somatosensory cortex (S1) $7 \mathrm{~d}$ after SCI. This reduction preceded an increase in connectivity between S1 and other cortical areas involved in nociceptive processing. In addition, VPL had increased connectivity to contralateral thalamus at 7 and $14 \mathrm{~d}$ after injury. The temporal pattern of the increase in functional connectivity within the thalamus and between cortical areas (particularly S1 and retrosplenial cortex) had a striking resemblance to the temporal pattern for the development of a "below-level" mechanical hypersensitivity in the same animals. Our findings suggest that below-level hypersensitivity is associated with functional disconnection (asynchrony) between the thalamus and cortical areas involved in nociceptive processing.
\end{abstract}

\section{Introduction}

Spinal cord injury (SCI) is associated with dramatic maladaptive neurological changes with catastrophic consequences, including the development of debilitating chronic neuropathic pain (SCI pain). The pain develops in $30-80 \%$ of patients with SCI and therefore it potentially affects millions of people worldwide and poses a significant clinical problem (Nepomuceno et al., 1979; Finnerup et al., 2001; Siddall et al., 2002). The pain is severe and unrelenting and mechanisms underlying its development are poorly understood (New et al., 1997; Siddall et al., 2003). It had been proposed, over a century ago, that pain due to injury of the spinal cord or the brain (central pain) results from abnormally suppressed inhibition in the thalamus (Head and Holmes, 1911). However, despite several breakthroughs in the field, the mechanisms for the engagement of inhibition, the source of the suppressed inhibition, and specific brain networks involved remain largely unknown (Boivie, 2005; Canavero and Bonicalzi, 2007).

\footnotetext{
Received June 20, 2012; revised Aug. 10, 2012; accepted Sept. 12, 2012.

Author contributions: R.P.G. and R.M. designed research; L.J., Y.J., and S.X. performed research; D.A.S., L.J., and R.M. analyzed data; D.A.S., S.X., and R.M. wrote the paper.

This research project was supported by a research grant from the National Institute of Neurological Disorders and Stroke (R01-NS069568 to R.M.), a research grant from the Department of Defense (SC090126 to R.M.), and an NIH Training Grant (T32-NS07375 to L.J.).

The authors declare no competing financial interests.

Correspondence should be addressed to Dr. Radi Masri, Department of Endodontics, Prosthodontics and Operative Dentistry, University of Maryland Dental School, 650 West Baltimore Street, 0ffice\#6253, Baltimore, MD 21201. E-mail: radi.masri@gmail.com.

DOI:10.1523/JNEUROSCI.2927-12.2012

Copyright $\odot 2012$ the authors $\quad 0270-6474 / 12 / 3215843-06 \$ 15.00 / 0$
}

Previous studies showed that SCI is associated with widespread changes along the neural axis that may result in SCI pain. In the spinal cord, these changes include axonal sprouting, enhanced neuronal activity and glial activation (Hulsebosch et al., 2009). SCI is associated with enhanced neuronal activity, increased blood oxygen level-dependent (BOLD) signals and neuronal reorganization in the cortex (Endo et al., 2008a,b; Quiton et al., 2010) and abnormalities in neuronal activity and glial function in the thalamus (Weng et al., 2003; Hains et al., 2006; Masri et al., 2009).

Some authors reported that SCI and deafferentation alter the firing properties of thalamic neurons, rendering them more likely to produce bursts of action potentials and suggested that this increased bursting results in neuropathic pain (Lenz et al., 1989; Wang and Thompson, 2008). Walton and Llinas (2010) reported that the increased bursting results in aberrant magnetoencephalographic (MEG) potential rhythms and they proposed that pain results from "thalamocortical dysrhythmia," whereby abnormalities in thalamic firing (bursting), caused by neuronal injury and deafferentation, disinhibit cortical structures responsible for pain perception (Walton and Llinas, 2010). However, the presence of thalamocortical dysrhythmia in SCI pain remains to be illustrated. To test the hypothesis that SCI leads to aberrant functional connectivity between the thalamus and the cortex, we performed a longitudinal resting-state fMRI (rsfMRI) study to assess changes in whole brain connectivity in a rat SCI model. 
a

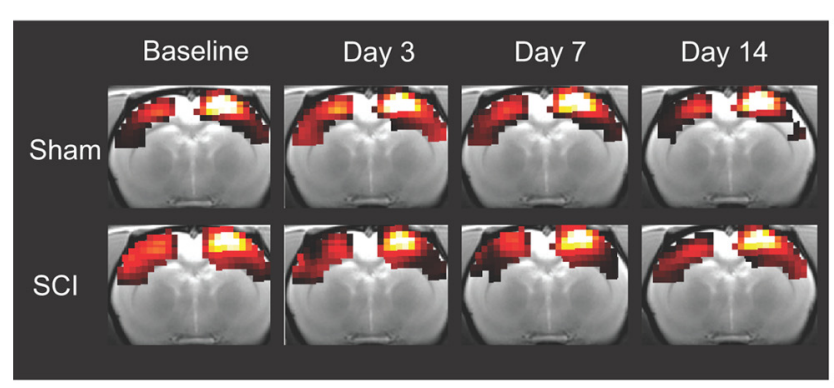

b

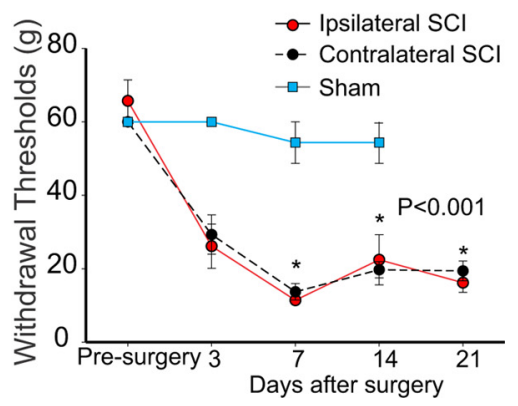

Figure 1. Reliability of brain imaging and behavioral assessment of spinal-lesioned and sham-operated animals. $\boldsymbol{a}$, The right hindpaw representation in S1HL seed network is shown for each group (top row, sham; bottom row, SCl) before surgery (baseline) and 3, 7, and $14 \mathrm{~d}$ after surgery. Images were thresholded at a $T$-value of 3 to highlight the extent of the network, and they were masked by the combined baseline scans for both groups. $\boldsymbol{b}$, Hindpaw (dorsal surface) mechanical withdrawal thresholds decrease over time and hypersensitivity develops bilaterally after $\mathbf{S C l}$. All values are means $\pm \mathrm{SE} ;{ }^{*} p<0.001$, statistically significant difference. Brain images are shown in neurological convention: left side of the image is left side of the brain.

\section{Materials and Methods}

SCI. All procedures were approved by our Institutional Animal Care and Use Committee and conducted in accordance with Animal Welfare Act regulations, PHS guidelines and the guidelines of the International Association for the Study of Pain. Fourteen adult female Sprague Dawley rats $(250-300 \mathrm{~g})$ were used. Animals were anesthetized with ketamine/ xylazine $(100 / 8 \mathrm{mg} / \mathrm{kg}$, i.p.). A midline, longitudinal incision $(10 \mathrm{~mm})$ overlaying C2-T2 area was made. The muscles were dissected under a dissecting microscope with blunt scissors to expose vertebrae C6 and C7. A laminectomy to expose the spinal cord was performed using rongeur and the dura mater covering the spinal cord was removed. A quartzinsulated platinum electrode ( $5 \mu \mathrm{m}$ tip) was targeted to the spinothalamic tract (STT) on the right side of the spinal cord rostral to C7 as described in Masri et al., (2009). Current (10 $\mu \mathrm{A}$ for $40 \mathrm{~s}$ ) was passed to produce an electrolytic lesion $\left(\sim 5 \mathrm{~mm}^{2}\right)$. Sham surgery was performed without performing laminectomy.

Behavior. Animals were tested on three consecutive days before SCI, at day $3,7,14$, and 21 postsurgery for the development of below-level hypersensitivity (relative to dermatome at which injury occurred). The animals were habituated for 2 weeks before behavioral testing. Calibrated von Frey filaments (Stoelting) were applied in ascending order to the dorsal surface of both hindpaws using the Ren (1999) method. Mechanical withdrawal threshold was defined as the force at which the animal withdrew to 3 of the 5 stimuli delivered. Repeated-measures (RM) ANOVA on Ranks was used to compare presurgery and postsurgery data in both the sham $(n=6)$ and SCI groups $(n=8)$. A $p$-value of $<0.05$ was considered significant.

fMRI. rsfMRI was used because it is a noninvasive imaging tool that can be repeated over time to assess changes in brain circuits within the same animal. rsfMRI experiments were performed on each animal in the sham and SCI groups before, and at 3, 7, and $14 \mathrm{~d}$ after surgery and additionally at $21 \mathrm{~d}$ after surgery for the SCI group. The scans were conducted immediately after assessing mechanical withdrawal thresholds. Animals were anesthetized with isoflurane (1.5\%). Respiration rate was monitored continuously throughout the experiment.

All MR experiments were performed on a Bruker BioSpec 70/30USR Avance III 7T scanner (Bruker Biospin MRI GmbH, Germany). Proton density-weighted images were obtained using a 2D RARE $(256 \times 256$ matrix, 14 coronal $1 \mathrm{~mm}$ slices, $3.5 \times 3.5 \mathrm{~cm}^{2}$ FOV) for anatomic reference. rsfMRI scans were acquired using an echo planar imaging (EPI) sequence (TR 1 s, $64 \times 64$ matrix, remaining geometry parameters identical to anatomic reference image, 550 volumes per scan).

Analysis. Because we had a particular interest in specific thalamic and cortical structures that receive inputs from the STT and exhibit abnormal neuronal activity following SCI [ventroposterior lateral thalamus (VPL), and hindpaw representation in the primary somatosensory cortex $(\mathrm{S} 1 \mathrm{HL})]$, we used seed-based analysis to assess how connectivity to these regions varies over time after SCI. Seeds were chosen based on anatomical locations in the Paxinos and Watson (1998) atlas. We first performed data preprocessing (slice timing correction, motion correction, normal- ization to a study-specific template, interpolation to voxel sizes of $0.5 \times$ $0.5 \times 0.5 \mathrm{~mm}$, bandpass filtering $(0.009-0.2 \mathrm{~Hz})$, and smoothing at 1 $\mathrm{mm}$ FWHM. We then extracted data from $0.3 \mathrm{~mm}$ radius spheres in the seed regions, and regressed these time courses with the signal at each voxel across the whole brain to reveal connectivity patterns for each animal. Motion parameters were included as nuisance variables. We did not remove global signal because correlation patterns and group differences become distorted after global signal regression (Saad et al., 2012). We did not include cardiac and respiratory factors in connectivity measures as regressors, but most of the cardiac and respiratory artifact is removed through the preprocessing steps we performed, particularly motion correction and motion regression in the first level analyses (Kalthoff et al., 2011). In addition, we performed independent component analysis to identify networks previously reported in resting-state rat networks at each time point to ensure that resting-state measures were reliable across time (data not shown). To illustrate the reliability of the resting-state networks in our dataset, in Figure $1 a$ we show networks from the seed region S1HL in both experimental groups at each time point.

Group analysis was performed using linear mixed models, with the connectivity maps as the dependent measures, animal as a random factor, and group and time as fixed factors. We examined the group-bytime-point interactions between connectivity at 3, 7, and $14 \mathrm{~d}$ postinjury compared with pre-injury between sham and SCI groups ( $21 \mathrm{~d}$ postinjury data were not included in the model, but were included in the plots). All preprocessing and analysis were performed in SPM8 (http://www.fil. ion.ucl.ac.uk/spm/). To correct for multiple comparisons, we used AFNI's AlphaSim (http://afni.nimh.nih.gov/afni/doc/manual/AlphaSim), which determined a cluster of 86 contiguous activated voxels at the $\alpha 0.05$ level would be significant. We extracted and plotted averaged adjusted $\beta$ values from significant clusters for each animal. To show change in connectivity over time, relative to pre-injury, the pre-injury time-point value for each cluster was subtracted from every other time-point value for the same animal. We also plotted relative connectivity between sham and SCI by subtracting the sham values from SCI. This allowed us to present changes in functional connectivity relative to both baseline values before surgery and shams in one plot.

\section{Results}

We and others have shown previously that the current SCI model results in bilateral mechanical hypersensitivity caudal (belowlevel) to the lesion site (Wang and Thompson, 2008; Davoody et al., 2011). Consistent with those studies, SCI animals developed bilateral mechanical hypersensitivity of the hindpaws that was significantly different ( $p<0.001$, RM ANOVA on Ranks) at 7 , 14 , and $21 \mathrm{~d}$ after SCI when compared with pre-injury thresholds and thresholds of sham animals (Fig. $1 b$ ).

Figure 2, $a$ and $d$, shows the change in connectivity of the right VPL seed (ipsilateral to SCI) in SCI relative to sham and to base- 
a

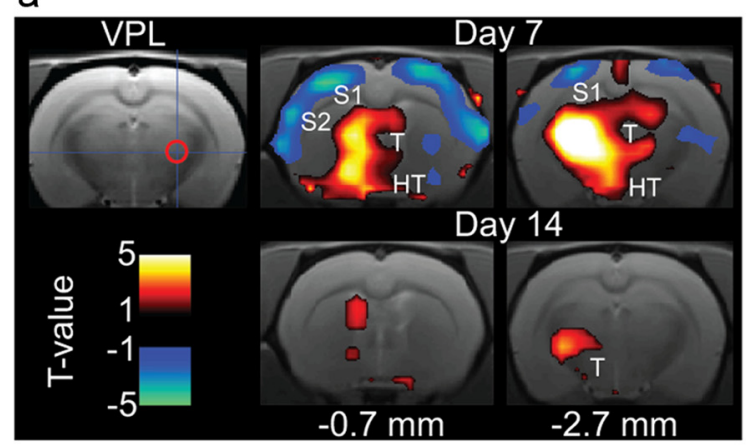

b

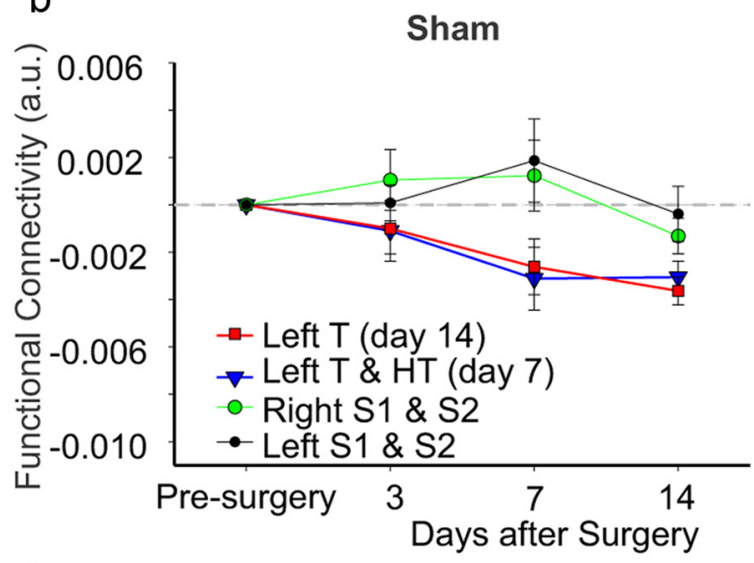

C
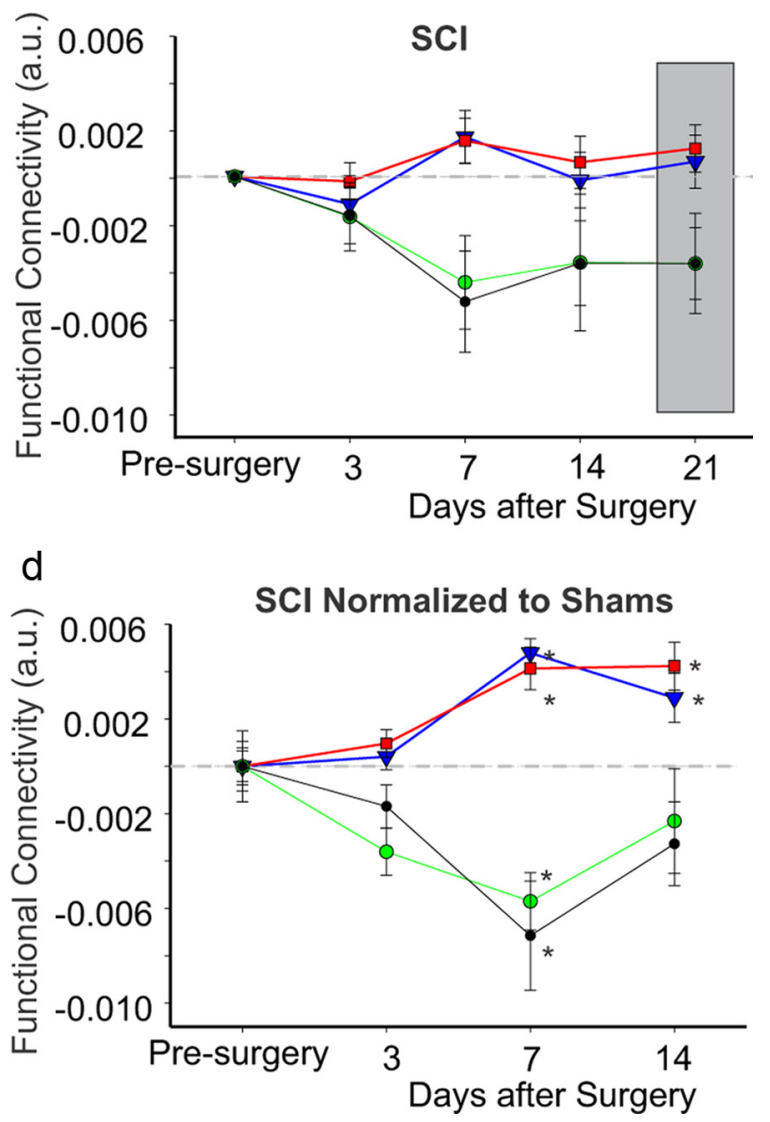

Figure 2. VPL functional connectivity over time. $\boldsymbol{a}$, Connectivity of the right VPL seed region (cross hair and red circle) and clusters that show increased (red) or decreased (blue) connectivity
Table 1. Summary of functional connectivity analysis

\begin{tabular}{|c|c|c|c|c|c|c|}
\hline \multirow{2}{*}{ Cluster location } & \multirow{2}{*}{$\begin{array}{l}\text { Clustersize } \\
\text { (\# of voxels) }\end{array}$} & \multirow{2}{*}{$\begin{array}{l}\text { Peak } \\
T \text {-value }\end{array}$} & \multirow{2}{*}{$\begin{array}{l}\text { Peak } \\
p \text {-value }\end{array}$} & \multicolumn{3}{|c|}{ Coordinates } \\
\hline & & & & $x$ & $y$ & $z$ \\
\hline \multicolumn{7}{|c|}{ Positive correlations with right VPL seed at day 7} \\
\hline \multirow[t]{7}{*}{ Left thalamus } & 1567 & 4.24 & $<0.001$ & -3.2 & -4.2 & 6.1 \\
\hline & & 4.10 & $<0.001$ & -1.6 & -7.7 & 2.6 \\
\hline & & 3.38 & 0.001 & 3.2 & -7.7 & 5.1 \\
\hline & & 3.01 & 0.002 & -1.1 & -0.2 & 9.6 \\
\hline & & 2.59 & 0.007 & -1.1 & 0.3 & 5.1 \\
\hline & & 2.40 & 0.010 & 1.6 & -4.7 & 2.6 \\
\hline & & 1.96 & 0.028 & 2.2 & -2.7 & 6.1 \\
\hline \multicolumn{7}{|c|}{ Negative correlations with right VPL seed at day 7} \\
\hline \multirow[t]{2}{*}{ Left S1/M1/S2/insula } & 147 & 3.61 & $<0.001$ & -3.8 & 0.8 & 2.1 \\
\hline & & 2.77 & 0.004 & -3.2 & -3.2 & 1.1 \\
\hline \multirow[t]{3}{*}{ Right S1/M1/S2/insula } & 178 & 2.70 & 0.005 & 4.3 & 2.8 & 3.1 \\
\hline & & 2.65 & 0.006 & 7.5 & -0.7 & 5.6 \\
\hline & & 2.58 & 0.007 & 3.2 & -0.7 & 1.1 \\
\hline \multicolumn{7}{|c|}{ Positive correlations with right VPL seed at day 14} \\
\hline \multirow[t]{5}{*}{ Left thalamus } & 826 & 4.23 & $<0.001$ & -2.7 & -7.7 & 2.6 \\
\hline & & 3.90 & $<0.001$ & 3.2 & -6.7 & 2.6 \\
\hline & & 3.70 & $<0.001$ & -3.8 & -3.2 & 6.6 \\
\hline & & 3.00 & 0.002 & 1.6 & -0.2 & 9.6 \\
\hline & & 2.69 & 0.005 & -1.6 & 1.3 & 5.6 \\
\hline \multicolumn{7}{|c|}{ Positive correlations with right $\mathrm{S} 1 \mathrm{HL}$ seed at day 7} \\
\hline \multirow[t]{2}{*}{ RSC } & 174 & 3.64 & $<0.001$ & -1.6 & -7.7 & 2.1 \\
\hline & & 2.72 & 0.005 & 0 & -2.7 & 0.6 \\
\hline \multicolumn{7}{|c|}{ Negative correlations with right S1HL seed at day 7} \\
\hline \multirow[t]{3}{*}{ Right S1, prelimbic } & 526 & 4.62 & $<0.001$ & 0.5 & 3.8 & 4.6 \\
\hline & & 3.91 & $<0.001$ & 4.3 & 0.8 & 2.1 \\
\hline & & 2.15 & 0.019 & -2.7 & 1.3 & 8.1 \\
\hline \multirow[t]{2}{*}{ Left thalamus } & 237 & 3.23 & 0.001 & -6.5 & -3.7 & 6.1 \\
\hline & & 2.84 & 0.003 & 0.5 & -3.7 & 8.1 \\
\hline \multirow[t]{2}{*}{ Left S1 } & 186 & 2.85 & 0.003 & -4.8 & 0.3 & 2.6 \\
\hline & & 2.27 & 0.014 & -3.8 & -3.7 & 1.1 \\
\hline \multicolumn{7}{|c|}{ Positive correlations with right S1HL seed at day 14} \\
\hline \multirow[t]{2}{*}{ Left S2, insula } & 93 & 3.78 & $<0.001$ & -7 & 0.3 & 6.1 \\
\hline & & 2.46 & 0.009 & -4.8 & 3.3 & 4.1 \\
\hline \multirow[t]{4}{*}{ RSC } & 404 & 3.50 & 0.001 & -1.6 & -7.7 & 2.1 \\
\hline & & 3.09 & 0.002 & 6.5 & -6.7 & 3.6 \\
\hline & & 3.08 & 0.002 & -5.9 & -7.7 & 4.1 \\
\hline & & 2.01 & 0.025 & -6.5 & -3.7 & 4.6 \\
\hline $\mathrm{ACC}$ & 109 & 3.16 & 0.001 & 0.5 & 3.3 & 1.6 \\
\hline
\end{tabular}

Peak voxels in significant clusters for right VPL and right S1HL seed analyses. Coordinates are from Paxinos and Watson (1998) atlas; $y$ is relative to bregma, $z=0$ is dorsal. HT, Hypothalamus; Ins, insula; M1, primary motor cortex; $\mathrm{S} 2$, second somatosensory cortex.

line (presurgery). There was no change $3 \mathrm{~d}$ postsurgery, but at 7 and $14 \mathrm{~d}$ connectivity was increased significantly in two clusters encompassing the contralateral thalamus ( $p$-values for peak voxels are provided in Table 1). Figure 2, $b$ and $c$, shows the change in connectivity over time in sham and SCI animals, respectively, for the clusters in Figure $2 a$. Figure $2 d$ shows the temporal pattern for the change in VPL connectivity for SCI relative to sham. The increased connectivity of the VPL seed to the contralateral thalamus (Fig. $2 d$ ) was strikingly similar to the temporal pattern for the development of hypersensitivity in the same animals (Fig. 1b).

relative to sham animals at days 7 and 14 postinjury. The sections in the current and following figure are referenced to Paxinos and Watson (1998) atlas in terms of distance to bregma. $\boldsymbol{b}$, Change in functional connectivity, relative to baseline, of the right VPL seed and the clusters shown in $\boldsymbol{a}$ at days 3,7 , and 14 after sham surgery. $\boldsymbol{c}$, Change in functional connectivity, relative to baseline, of the right VPL seed and the clusters shown in $\boldsymbol{a}$ at days 3, 7, 14, and 21 after SCI. Gray box is used to highlight day 21 results. $\boldsymbol{d}$, Plot of all clusters (shown in $\boldsymbol{a}$ ) with increased or decreased functional connectivity at all time points in SCI relative to sham animals (gray line) and pre-injury. For full details on specific clusters, refer to Table 1. * Significant $(p<0.05$, cluster-corrected). Error bars represent SEM. a.u., Arbitrary units, HT, hypothalamus; $\mathrm{S} 2$, second somatosensory cortex; $T$, thalamus. 
a

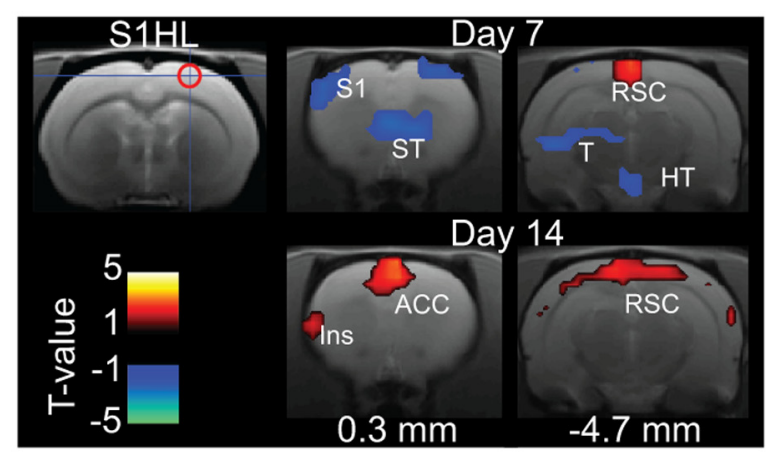

b

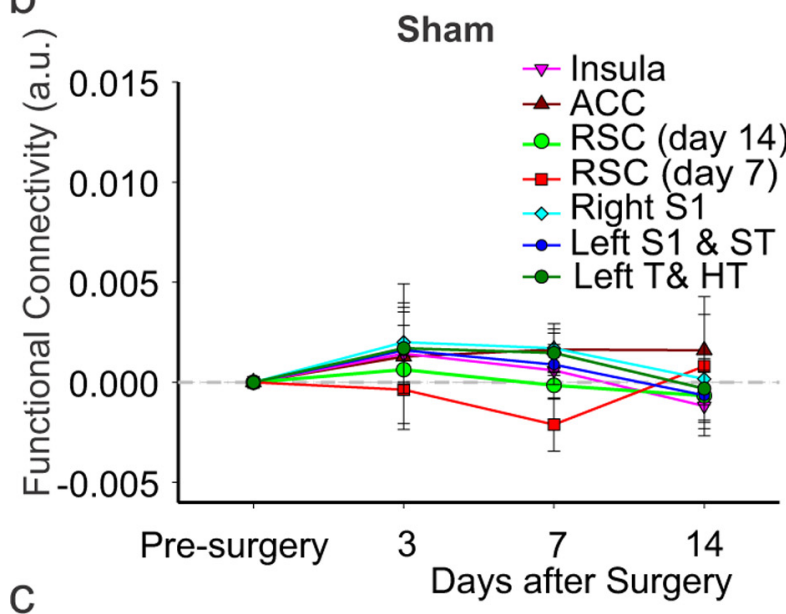

C

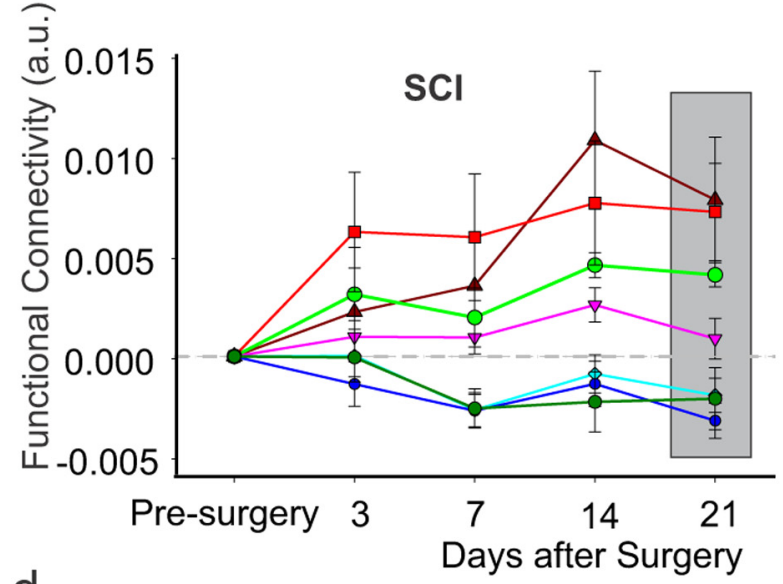

$\mathrm{d}$

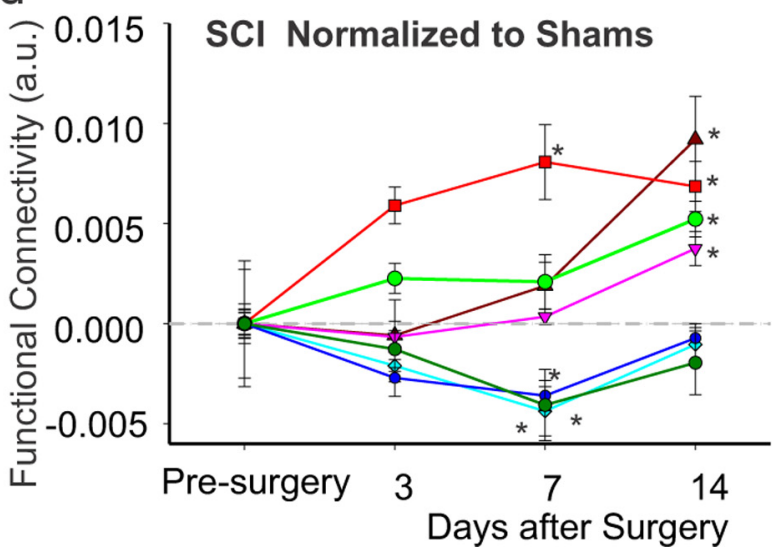

These findings suggest that the increase in connectivity within the thalamus is associated with the development of hypersensitivity in SCI animals. The VPL had increased connectivity to other areas including midline thalamic nuclei, periaqueductal gray and hippocampus. In SCI, VPL had reduced connectivity to large cortical areas that encompassed the bilateral S1 (Fig. 2a). However, unlike the increased connectivity in the thalamus, the temporal pattern of the reduced connectivity was transient, being significant at day 7 after SCI, but not at later time points (Fig. $2 d$ ). Thus, the reduced connectivity did not track the development of hypersensitivity (Fig. 1b). These findings suggest that lesions of the ventrolateral portion of the spinal cord are associated with a significant reduction in coherent rhythmic oscillations or disconnection between the thalamus and the cortex. We examined connectivity of left VPL and obtained similar results to those of right VPL (data not shown).

For the right S1HL seed, connectivity in SCI was significantly altered in 7 clusters that encompassed several areas in the thalamus and cortex (Fig. $3 a$ ). In 3 clusters, including contralateral thalamus and bilateral S1, connectivity was reduced at $7 \mathrm{~d}$ after injury (Fig. 3a). In the remaining 4 clusters, connectivity to the S1HL seed was increased. These areas included the insula, the anterior cingulate cortex (ACC) and the retrosplenial cortex (RSC) (Fig. 3a). Examination of changes in connectivity in sham animals revealed little changes over time (Fig. $3 b$ ). However, in SCI connectivity of S1HL appeared to increase over time up to $21 \mathrm{~d}$ (Fig. 3c). The increase in connectivity of the S1HL to the insula and ACC (Fig. 3a) was only evident at day 14 after SCI while connectivity to RSC was significantly increased at days 7 and 14 (Fig. 3a,d) and more faithfully mimicked the development of hypersensitivity after SCI. We performed the same analysis on the left S1HL and obtained similar results (data not shown).

\section{Discussion}

In a rat model of SCI we found decreased functional connectivity between the thalamus (VPL) and the cortex (S1) $7 \mathrm{~d}$ after SCI. This reduction preceded an increase in connectivity between $\mathrm{S} 1$ and several cortical areas (insula, ACC, and RSC) implicated in nociceptive processing. The temporal pattern of the increase in functional connectivity within the thalamus and between cortical areas (particularly S1 and RSC) had a striking resemblance to the temporal pattern for the development of below-level, mechanical hypersensitivity in the same animals.

Our findings suggest that SCI is associated with a functional disconnection (asynchrony) between the thalamus and cortex. MEG studies in chronic pain patients suggested that thalamic neural activity is abnormally enhanced and firing occurs synchronously at a low frequency $(<5 \mathrm{~Hz})$ (Walton and Llinas, 2010). These low-frequency oscillations have been shown to release neurons in the cortex from inhibition (Leznik et al., 2002), which in turn will cause cortical neurons to discharge in sponta-

$\leftarrow$

Figure 3. S1HL functional connectivity over time. $\boldsymbol{a}$, Connectivity of the right S1HL seed region (cross hair and red circle) and clusters that show increased (red) or decreased (blue) connectivity relative to sham animals at days 7 and 14 postinjury. $\boldsymbol{b}$, Change in functional connectivity, relative to baseline, of the right $\mathrm{S1HL}$ seed and the clusters shown in $\boldsymbol{a}$ at days 3,7 , and 14 after sham injury. $c$, Change in functional connectivity, relative to baseline, of the right S1HL seed and the clusters shown in $\boldsymbol{a}$ at days 3, 7, 14, and 21 after $\mathrm{SCl}$. Gray box is used to highlight day 21 results. $\boldsymbol{d}$, Plot of all the clusters in $\boldsymbol{a}$ showing increased or decreased connectivity at all time points in $\mathrm{SCl}$ animals relative to sham (gray line) and pre-injury. For cluster details refer to Table 1. *Significant ( $p<0.05$, cluster-corrected). Error bars represent SEM. Ins, Insula; ST, striatum. See Figure 2 for other abbreviations. 
neous, continuous oscillations at a higher frequency (Llinás et al., 2005). Although BOLD signal fluctuations occur at a much slower rate $(<0.2 \mathrm{~Hz})$ than MEG signals, we propose that similar mechanisms may underlie the abnormalities in cortical oscillations observed in this study. In the same model of SCI used here, spontaneous activity and incidence of bursting in VPL were abnormally increased and the bursts occurred at a low frequency $<0.4 \mathrm{~Hz}$ and can therefore lead to reduced inhibition in cortical areas involved in nociceptive processing (Wang and Thompson, 2008). Indeed, we reported previously that neuronal activity and the percentage of bursting cells in S1 are significantly increased (Quiton et al., 2010). However, SCI is associated with massive changes in spinal and supraspinal structures. Therefore, the change in connectivity may not only be due to deafferentation and abnormal bursting in the thalamus, but could also be due to increased afferent activity in the spinal cord (Christensen and Hulsebosch, 1997; Vierck et al., 2000) or indirectly due to glial activation in spinal and supraspinal structures (Hulsebosch et al., 2009). These spinal and supraspinal changes (reported in models of SCI different from the one we used) may result in altered thalamocortical rhythms and changes in connectivity. It remains to be seen whether the changes in connectivity reported here also occur in other SCI models and in patients with SCI pain.

It is possible that the changes in connectivity may not relate to SCI and the development of hypersensitivity, but rather to changes in the integrity of supraspinal pathways that occur after injury. SCI causes anatomical changes in brain circuitry (Gustin et al., 2010; Henderson et al., 2011) and chemistry (Stanwell et al., 2010) that alter neuronal activity in supraspinal structures (Morrow et al., 2000; Paulson et al., 2005; Endo et al., 2008a; Endo et al., 2008b).

A concern in rodent $\mathrm{fMRI}$ studies is the anesthetic used. We used isoflurane because it maintains the topological features of resting-state networks observed in the awake condition (Liang et al., 2012) and because it allowed us to perform repeated rsfMRI without the confounding effects of movement and stress. However, isoflurane anesthesia may attenuate functional connectivity (Becerra et al., 2011) and may have lingering effects on cerebral blood flow when administered repeatedly (Wegener and Wong, 2008). In sham animals functional connectivity of the VPL to two clusters in the contralateral thalamus appeared to decrease over time (Fig. 2b) suggesting that longitudinal fMRI scans affect resting state networks, which could be an effect of repeated isoflurane administration. Because of this, we compared changes in connectivity in SCI and sham at every time point. However, inherent to this analysis is the assumption that anesthesia exerts similar effects on both groups. Nonetheless, in the majority of clusters connected to VPL and S1HL seeds, there was little change over time in shams (Figs. $2 b, 3 b$ ), suggesting that connectivity changes are driven by changes in the SCI group.

Although the animal model used in this study (STT injury, see also Vierck and Light, 1999) may appear to be less relevant clinically than other SCI models (e.g., Siddall et al., 1995), it has several advantages. It is associated with less variability because of the controlled size, location, and extent of the lesion; it is associated with less morbidity, so early time points after surgery can be studied; and the lesion spares the motor fibers and does not result in paralysis or motor deficits and therefore the changes we observe in functional connectivity are less likely to be confounded by injury to motor fibers in the spinal cord. In this model, we found bilateral changes in functional connectivity, even though the injury was unilateral. This finding is not surprising, since resting-state networks are almost always bilateral, especially in the rat (Becerra et al., 2011).

In addition, we find that animals develop bilateral mechanical hypersensitivity (discussed in: Davoody et al., 2011; Lucas et al., 2011). The development of hypersensitivity was associated with changes in functional connectivity. However, we did not test this relationship directly because changes in connectivity were assessed when the animals were anesthetized, while hypersensitivity was determined using evoked responses in awake animals. Additionally, the use of reflexive behavioral methods (mechanical withdrawal thresholds) to characterize pain may be problematic in animals with SCI (Baastrup et al., 2010). In the future it will be useful to use operant tests that reveal the presence of spontaneous pain (Davoody et al., 2011) and correlate the findings with changes in functional connectivity in awake animals after SCI.

\section{References}

Baastrup C, Maersk-Moller CC, Nyengaard JR, Jensen TS, Finnerup NB (2010) Spinal-, brainstem- and cerebrally mediated responses at- and below-level of a spinal cord contusion in rats: evaluation of pain-like behavior. Pain 151:670-679.

Becerra L, Pendse G, Chang PC, Bishop J, Borsook D (2011) Robust reproducible resting state networks in the awake rodent brain. PLoS One 6:e25701.

Boivie J (2005) Central pain. In: Wall and Melzack's textbook of pain (McMahon S, Koltzenburg M, eds), pp 1057-1074. Oxford: Churchill Livingstone.

Canavero S, Bonicalzi V (2007) Central pain syndrome: elucidation of genesis and treatment. Expert Rev Neurother 7:1485-1497.

Christensen MD, Hulsebosch CE (1997) Chronic central pain after spinal cord injury. J Neurotrauma 14:517-537.

Davoody L, Quiton RL, Lucas JM, Ji Y, Keller A, Masri R (2011) Conditioned place preference reveals tonic pain in an animal model of central pain. J Pain 12:868-874.

Endo T, Spenger C, Hao J, Tominaga T, Wiesenfeld-Hallin Z, Olson L, Xu XJ (2008a) Functional MRI of the brain detects neuropathic pain in experimental spinal cord injury. Pain 138:292-300.

Endo T, Spenger C, Westman E, Tominaga T, Olson L (2008b) Reorganization of sensory processing below the level of spinal cord injury as revealed by fMRI. Exp Neurol 209:155-160.

Finnerup NB, Johannesen IL, Sindrup SH, Bach FW, Jensen TS (2001) Pain and dysesthesia in patients with spinal cord injury: a postal survey. Spinal Cord 39:256-262.

Gustin SM, Wrigley PJ, Henderson LA, Siddall PJ (2010) Brain circuitry underlying pain in response to imagined movement in people with spinal cord injury. Pain 148:438-445

Hains BC, Saab CY, Waxman SG (2006) Alterations in burst firing of thalamic VPL neurons and reversal by $\mathrm{Na}(\mathrm{v}) 1.3$ antisense after spinal cord injury. J Neurophysiol 95:3343-3352.

Head H, Holmes G (1911) Sensory disturbances from cerebral lesions. Brain 34:102-254.

Henderson LA, Gustin SM, Macey PM, Wrigley PJ, Siddall PJ (2011) Functional reorganization of the brain in humans following spinal cord injury: evidence for underlying changes in cortical anatomy. J Neurosci 31:26302637.

Hulsebosch CE, Hains BC, Crown ED, Carlton SM (2009) Mechanisms of chronic central neuropathic pain after spinal cord injury. Brain Res Rev 60:202-213

Kalthoff D, Seehafer JU, Po C, Wiedermann D, Hoehn M (2011) Functional connectivity in the rat at 11.7T: impact of physiological noise in resting state fMRI. Neuroimage 54:2828-2839.

Lenz FA, Kwan HC, Dostrovsky JO, Tasker RR (1989) Characteristics of the bursting pattern of action potentials that occurs in the thalamus of patients with central pain. Brain Res 496:357-360.

Leznik E, Makarenko V, Llinás R (2002) Electrotonically mediated oscillatory patterns in neuronal ensembles: an in vitro voltage-dependent dyeimaging study in the inferior olive. J Neurosci 22:2804-2815.

Liang Z, King J, Zhang N (2012) Intrinsic organization of the anesthetized brain. J Neurosci 32:10183-10191.

Llinás R, Urbano FJ, Leznik E, Ramírez RR, van Marle HJF (2005) Rhythmic 
and dysrhythmic thalamocortical dynamics: GABA systems and the edge effect. Trends Neurosci 28:325-333.

Lucas JM, Ji Y, Masri R (2011) Motor cortex stimulation reduces hyperalgesia in an animal model of central pain. Pain 152:1398-1407.

Masri R, Quiton RL, Lucas JM, Murray PD, Thompson SM, Keller A (2009) Zona incerta: a role in central pain. J Neurophysiol 102:181-191.

Morrow TJ, Paulson PE, Brewer KL, Yezierski RP, Casey KL (2000) Chronic, selective forebrain responses to excitotoxic dorsal horn injury. Exp Neurol 161:220-226.

Nepomuceno C, Fine PR, Richards JS, Gowens H, Stover SL, Rantanuabol U, Houston R (1979) Pain in patients with spinal cord injury. Arch Phys Med Rehabil 60:605-609.

New PW, Lim TC, Hill ST, Brown DJ (1997) A survey of pain during rehabilitation after acute spinal cord injury. Spinal Cord 35:658-663.

Paulson PE, Gorman AL, Yezierski RP, Casey KL, Morrow TJ (2005) Differences in forebrain activation in two strains of rat at rest and after spinal cord injury. Exp Neurol 196:413-421.

Paxinos G, Watson C (1998) The rat brain in stereotaxic coordinates. San Diego: Academic.

Quiton RL, Masri R, Thompson SM, Keller A (2010) Abnormal activity of primary somatosensory cortex in central pain syndrome. J Neurophysiol 104:1717-1725.

Ren K (1999) An improved method for assessing mechanical allodynia in the rat. Physiol Behav 67:711-716.

Saad ZS, Gotts SJ, Murphy K, Chen G, Jo HJ, Martin A, Cox RW (2012) Trouble at rest: how correlation patterns and group differences become distorted after global signal regression. Brain Connect 2:25-32.

Siddall PJ, Yezierski RP, Loeser JD (2002) Taxonomy and epidemiology of spinal cord injury pain. In: Progress in pain research and management. (Yezierski RP, Burchiel KJ, eds), pp 9-24. Seattle: IASP.
Siddall PJ, McClelland JM, Rutkowski SB, Cousins MJ (2003) A longitudinal study of the prevalence and characteristics of pain in the first 5 years following spinal cord injury. Pain 103:249-257.

Siddall P, Xu CL, Cousins M (1995) Allodynia following traumatic spinal cord injury in the rat. Neuroreport 6:1241-1244.

Stanwell P, Siddall P, Keshava N, Cocuzzo D, Ramadan S, Lin A, Herbert D, Craig A, Tran Y, Middleton J, Gautam S, Cousins M, Mountford C (2010) Neuro magnetic resonance spectroscopy using wavelet decomposition and statistical testing identifies biochemical changes in people with spinal cord injury and pain. Neuroimage 53:544-552.

Vierck CJ Jr, Siddall P, Yezierski RP (2000) Pain following spinal cord injury: animal models and mechanistic studies. Pain 89:1-5.

Vierck CJ Jr, Light AR (1999) Effects of combined hemotoxic and anterolateral spinal lesions on nociceptive sensitivity. Pain 83:447-457.

Walton KD, Llinas RR (2010) Central pain as a thalamocortical dysrhythmia: a thalamic efference disconnection? In: Translational pain research: from mouse to man (Kruger L, Light AR, eds). Boca Raton, FL: Taylor and Francis Group.

Wang G, Thompson SM (2008) Maladaptive homeostatic plasticity in a rodent model of central pain syndrome: thalamic hyperexcitability after spinothalamic tract lesions. J Neurosci 28:11959-11969.

Wegener S, Wong EC (2008) Longitudinal MRI studies in the isofluraneanesthetized rat: long-term effects of a short hypoxic episode on regulation of cerebral blood flow as assessed by pulsed arterial spin labelling. NMR Biomed 21:696-703.

Weng HR, Lenz FA, Vierck C, Dougherty PM (2003) Physiological changes in primate somatosensory thalamus induced by deafferentation are dependent on the spinal funiculi that are sectioned and time following injury. Neuroscience 116:1149-1160. 